\title{
CALCULATION OF PRESSURE DROP IN NARROW ROTATING ANNULAR CLEARANCES
}

\section{Y. Nakashima, S. Oliveira Jr. ${ }^{a}$ and E. F. Caetano \\ ${ }^{a}$ University of São Paulo \\ Polytechnic School \\ Mechanical Engineering Dep. Av. Prof. Mello Moraes, 2231 \\ Cidade Universitária \\ CEP 05508-900 - São Paulo, SP - Brazil celyukio@usp.br, silvio.oliveira@poli..usp.br \\ ${ }^{\mathrm{b}}$ PETROBRAS - R\&D Center (CENPES) Ilha do Fundao - Quadra 7 CEP 21949-900 - Rio de Janeiro, RJ - Brazil ecaetano@petrobras.cenpes.br}

\section{ABSTRACT}

This paper presents a review of correlations used for pressure drop calculation in narrow annular clearances with rotation of inner cylinder. Based on these review, a new general correlation is proposed to calculate pressure drop through such clearances. Results are compared with experimental data for different flow regimes: laminar, laminar with Taylor vortex, smooth and rough turbulent, with good agreement. The main objective is to develop a simple and general correlation that could be used for backflow calculation in twin-screw multiphase pumps. In this kind of pump, the volumetric efficiency is defined mainly by the backflow rate which occurs in the annular clearance between screw and casing. Usually, the backflow is calculated ignoring the influence of shaft rotation over pressure drop and does not consider the several possible flow regimes. Depending on operational conditions, this simplification can lead to significant errors.

Keywords: annular channel, pressure drop, torque coefficient, rotating inner, screw pumps.

\section{NOMENCLATURE}

\section{Latin Symbols}

$\mathbf{C}_{\mathbf{M}}$ torque coefficient

$\mathbf{D}_{\mathbf{h}} \quad$ hydraulic diameter (m)

e eccentricity (m)

Fg geometric factor

$\mathbf{k}_{\text {an }}$ correction coefficient for flow in annulus

$\mathbf{k}_{\varepsilon} \quad$ correction coefficient for eccentricity

$\mathbf{k}_{\text {rot }}$ correction coefficient for rotation

$\mathbf{k}_{\mathrm{r}}$ roughness $(\mathrm{m})$

l length (m)

P pressure (bar)

$\mathbf{R}$ radius (m)

Re Reynolds number $(\operatorname{Re}=\rho U 2 s / \mu)$

s channel height $(\mathrm{m})$

t time (s)

Ta Taylor number $\left(T a=\rho \omega R_{i}{ }^{1 / 2} s^{3 / 2} / \mu\right)$

$\mathbf{U}$ average axial velocity $(\mathrm{m} / \mathrm{s})$

\section{Greek Symbols}

$\beta \quad$ experimental parameter in Eq. (3)

$\Delta$ difference

$\varepsilon \quad$ relative eccentricity

$\lambda$ axial friction coefficient

$\mu \quad$ dynamic viscosity (Pa.s)

$v \quad$ kinematic viscosity $\left(\mathrm{m}^{2} / \mathrm{s}\right)$

$\rho \quad$ specific mass $\left(\mathrm{kg} / \mathrm{m}^{3}\right)$

$\omega$ angular velocity ( $\mathrm{rad} / \mathrm{s}$ )

\section{Subscripts}

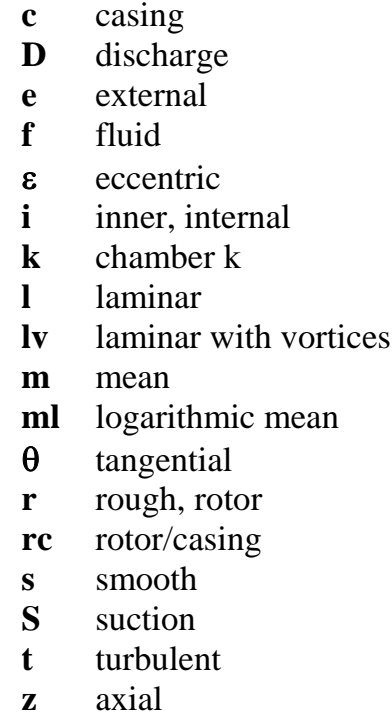

\section{Superscripts}

- $\quad$ quantity per time unit (1/s)

\section{INTRODUCTION}

The evaluation of backflow rate in screw pumps depends fundamentally on the comprehension of the flow in annular channels in which the inner cylinder rotates. In screw pumps, the pressure difference between two consecutive chambers forces part of the working fluid through the peripheral clearance back to the suction region as shown in Fig. (1). Since the 
geometry of this clearance recalls the geometry of narrow annular channels with rotation of inner cylinder, then the correct understanding of the phenomena involved in this kind of channel flow becomes extremely important when calculating pump performance. Besides, the required torque to move the inner cylinder is necessary for calculation of viscous losses in the pump periphery and, therefore, to correctly evaluate pump shaft power.

The work described in this paper aimed a literature review and - based on this - a development of a general correlation to calculate friction coefficient and torque coefficient in the cited flow. The need of such correlation raised during the development of a thermo-hydraulic model of a twinscrew multiphase pump (see Nakashima, Oliveira and Caetano (2002)). This kind of pump, which is been prototyped by Petrobras, is able to transport unprocessed petroleum (i.e. gas-liquid mixtures). Several models tried to calculate the backflow rate through the peripheral clearances using correlations for static annular channels. However, as it will be described in next items, the rotation of inner cylinder can have a significant influence on axial flow.

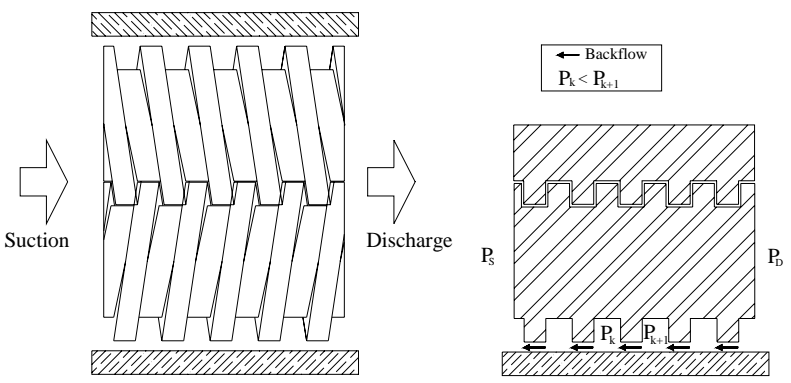

Figure 1. Backflow in the peripheral clearance of screw-pumps.

\section{FLOW IN ANNULAR CHANNELS WITH ROTATION OF INNER CYLINDER}

\section{Introduction}

Generically, the flow between concentric cylinders with rotation of inner one can be considered a composition of three basic flows: Couette, Poiseuille and Taylor, which can be either laminar or turbulent. As illustrated in Fig. (2), the Couette flow is caused by rotation of inner cylinder and the Poiseuille flow takes place due to pressure difference between channel inlet and outlet. Taylor flow appears due to centrifugal forces after a critical rotation and is characterized by a sequence of toroidal vortexes. They are distributed tangentially and have alternate senses.

Kaye and Elgar (1957) show experimentally that, depending on axial and tangential velocities, these basic flows will form four different regimes: laminar, laminar with Taylor vortexes, turbulent and turbulent with Taylor vortexes (see Fig. (3a)). When the flow is laminar, the axial and tangential components of the fluid velocity are independent of each other. In this case, it is clear that rotation will not influence axial friction losses. On the other hand, when the Taylor vortexes appear in the flow due to centrifugal forces - after a critical rotation - and when the flow is turbulent, the velocity components are not independent anymore. Consequently, in these regimes the rotation of the inner cylinder is expected to influence axial friction losses. Actually, several authors confirmed the influence of rotation over axial friction coefficient $(\lambda)$. Figure (3b), for instance, shows experimental results from Yamada (1962) where there is clearly an increase on the friction coefficient as rotation, represented by Taylor number $\left(T a=\rho \omega R_{i}^{1 / 2} s^{3 / 2} / \mu\right)$ is raised. In this figure, horizontal lines represent friction coefficient values obtained with usual channel flow correlations. When the flow is laminar ( $\mathrm{Re}=1000$ in Fig. (3b)) there is a sudden increase at a certain critical tangential velocity. At this point - that corresponds to the appearance of Taylor vortexes - the velocity components abruptly stop being independent of each other and the rotation of inner cylinder becomes important. For turbulent flow, velocity components are always dependent on each other and, therefore, the rotation influence is smoother than in laminar case. However, if axial velocity is large, the influence of rotation can only be noticed at high tangential velocities.

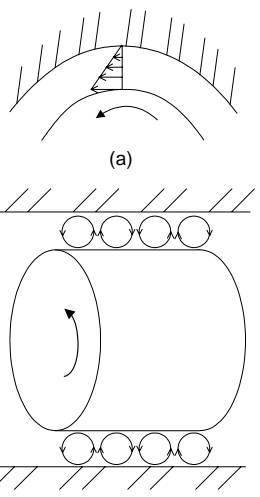

(b)
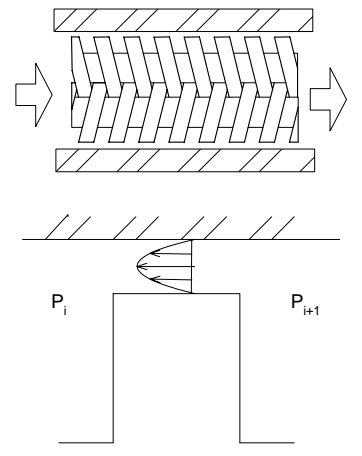

(c)
Figure 2. Schematic illustration of possible flow types inside annular channels with rotation of inner

cylinder: a) Couette flow, b) Taylor flow and c) Poiseuille flow.

\section{Pressure drop}

The relation between pressure drop and axial mean velocity in a channel can be given by:

$$
\Delta P=\frac{\rho U_{z}^{2}}{2}\left(\frac{\lambda l}{2 s}\right)
$$

According to Idelchik (1994), the friction coefficient in narrow static annular channels for laminar $\left(\lambda_{l}\right)$, smooth $\left(\lambda_{s}\right)$ and rough $\left(\lambda_{r}\right)$ turbulent flows can be calculated respectively by: 


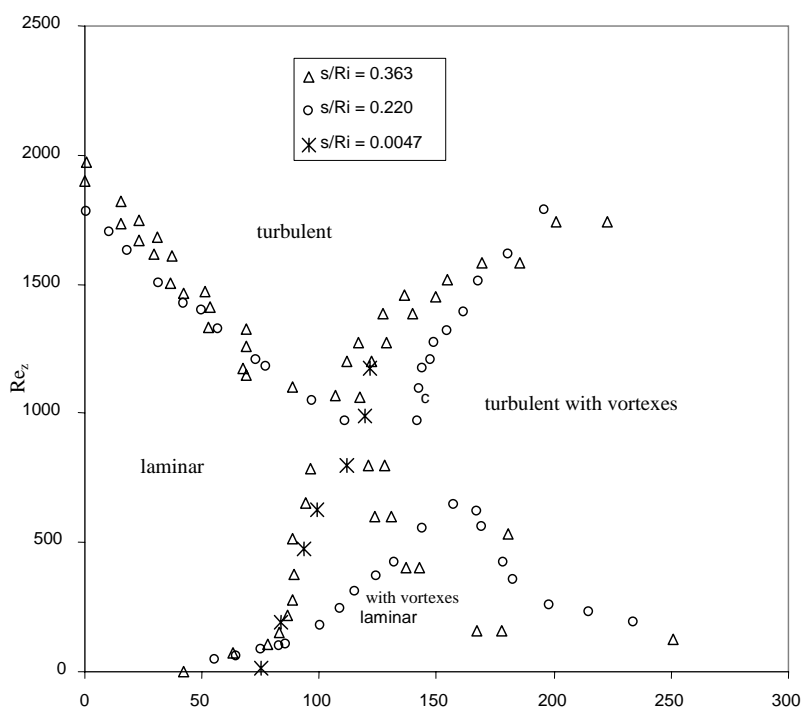

(a) Kaye and Elgar (1957)

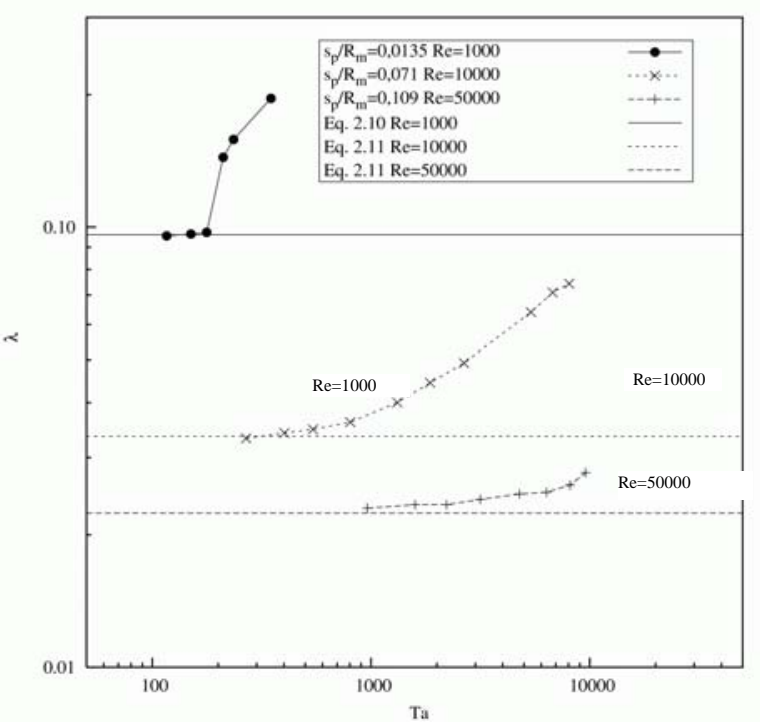

(b) Yamada (1962)

Figure 3. Flow regimes (after Kaye and Elgar (1957)) and behavior of friction coefficient (after Yamada (1962)) between two concentric cylinders with rotation of the inner one and axial flow.

$$
\begin{aligned}
& \lambda_{l}=\frac{96}{R e_{z}}, \lambda_{s}=\frac{0.3354}{R e_{z}^{0.25}} \text { and } \\
& \lambda_{r}=\left(2 \log \left(\frac{s}{k_{r}}\right)+1.74\right)^{-2}
\end{aligned}
$$

However, as the inner cylinder starts rotating, the tangential component of fluid velocity will influence the flow. In this case, Eqs. (2) will not be valid anymore. Therefore, when the flow is not laminar, the friction coefficient must be a function of both axial and tangential velocities. The first attempt to include rotation effects in the calculation of friction coefficient was made by Suzuki (1929). Assuming the $1 / 7^{\text {th }}$ power law valid near the wall either in the axial or tangential directions, he developed the following correlation for smooth turbulent flow:

$$
\begin{aligned}
\lambda_{s}= & \frac{0.3216}{\operatorname{Re}_{z}^{1 / 4}} \cdot \frac{1}{2}\left\{\left[1+0.629\left(\frac{\omega R_{i}}{U_{z}}\right)^{2}\right]^{3 / 8}+\right. \\
& \left.+\left[1+0.629\left(\beta \frac{\omega R_{i}}{U_{z}}\right)^{2}\right]^{3 / 8}\right\}
\end{aligned}
$$

Where $\beta$ is a parameter determined experimentally. Equation (3) can be directly compared to the Blasius equation for friction coefficient in smooth turbulent flow. Despite a slight difference in the coefficient (0.3216 in Eq. (3) and 0.3354 in Blasius equation for narrow annular channels) Eq. (3) could be considered as the Blasius equation with a correction factor for rotational influence over axial pressure drop.

Yamada (1962) proposed a correlation (Eq. (4)) using a similar approach and made extensive experimental measurements to determine friction coefficient in several operational conditions and geometries. The comparison of his experimental data with Eqs. (3) and (4) showed that the values obtained with Eq. (4) have a good agreement in the turbulent region. In the laminar with Taylor vortexes region, the correlation shows poor agreement. It is possible to compare Eqs. (3) and (4) and realize that they are quite similar when $\beta=1.0$.

$$
\lambda_{s}=\frac{0.3071}{R e_{z}^{0.24}}\left[1+\left(\frac{7}{16}\right)^{2}\left(\frac{\omega R_{i}}{U_{z}}\right)^{2}\right]^{0.38}
$$

Other factor that can influence pressure drop and mean axial flow is the eccentricity between inner and outer cylinders. In screw-pumps operation, such eccentricity is expected to be caused either by design requirements or screw-shaft bending.

For the non-rotating cases, the eccentricity will cause a reduction in pressure drop and an increase in axial flow as explained in Caetano, Shohan and Brill (1992) and Gunn and Darling (1963), respectively, for laminar and turbulent cases. When the flow is laminar, Eq. (5) can be analytically found for narrow $\left(\mathrm{s}_{\mathrm{p}} / \mathrm{R}_{\mathrm{i}} \approx 1\right)$ eccentric channels:

$$
\lambda_{\varepsilon, l}=\frac{\lambda_{l}}{\left(1+1.5 \varepsilon^{2}\right)}
$$

In the case of narrow channels with turbulent flow, many correlations were proposed by different researchers (Tao and Donovan (1953), Gunn and Darling (1963), Wincek (1992) and Idelchik (1994)). The comparison between the results for each equation in Fig. (4) shows similar results. Also in this figure, the results of Gunn and Darling (1963) equation for $\operatorname{Re}=3000$ and $\operatorname{Re}=100000$ evidence that the magnitude of axial Reynolds number has little 
importance over friction coefficient. Therefore, the pressure drop reduction can be considered as a function of eccentricity only as in the laminar case. In this paper, only the equation of Tao and Donovan (1955) will be presented because it can be used either for smooth or rough turbulent flow:

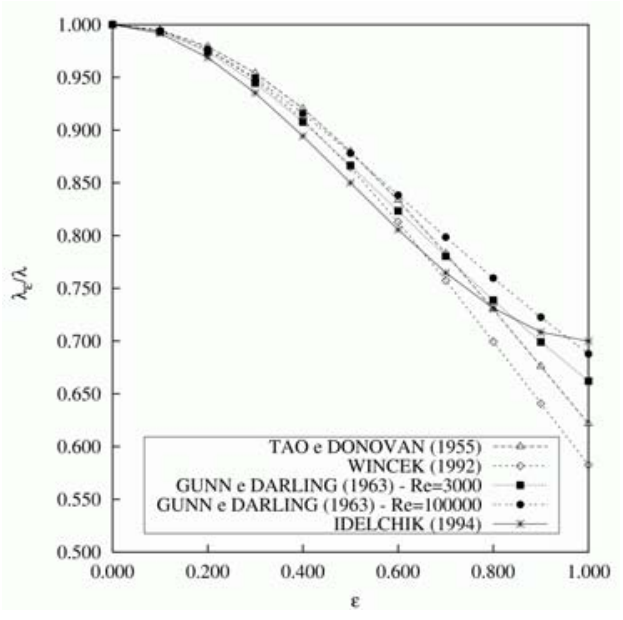

Figure 4. Reduction in friction coefficient due to eccentricity in turbulent flow according to several authors.

$$
\begin{gathered}
\lambda_{\varepsilon, t}=\lambda_{t}\left[\frac{1}{\pi} \int_{0}^{\pi}(1+\varepsilon \cdot \cos \phi)^{\frac{3}{2-n}} d \phi\right]^{-(2-n)}, \\
n=\left\{\begin{array}{cc}
0.25 \text { ( smooth turbulent) } \\
0 \quad \text { (rough turbulent) }
\end{array}\right.
\end{gathered}
$$

When the inner cylinder rotates, the influence of eccentricity is similar to that found in static cases if narrow channels are considered. Ooms and Kampman-Reinhartz (1996) and Escudier et al. (2000) studied numerically and experimentally the influence of eccentricity in channels with inner cylinder rotation and laminar flow. They found that when there is rotation, the influence of eccentricity is different from the static case: friction coefficient increase is higher. However, as the relation between internal and external radii approaches 1.0, the results of rotating and static cases become almost the same. When the flow is turbulent, Tao and Donovan (1955) and Nouri and Whitelaw (1997) found that the influence of eccentricity is almost the same in rotating and static cases.

\section{Torque}

The studies about torque between two cylinders with rotation of the inner one usually consider only the case without axial flow and are shown below. The influence of axial flow over the needed torque to move the inner cylinder, that is expected when the velocity components are not independent of each other, was not found in the literature review.
For laminar flow, the torque coefficient for concentric and eccentric cases can be calculated using the equation developed analytically by Diprima and Stuart (1972) for narrow channels:

$$
C_{M \varepsilon, l}=\frac{T}{\frac{1}{2} \pi \rho \omega^{2} R_{i}^{4} B}=\frac{4}{R e_{\theta}}\left\{\frac{2\left(1+2 \varepsilon^{2}\right)+3 s_{p} / R_{i}}{\left(1-\varepsilon^{2}\right)^{1 / 2}\left(2+\varepsilon^{2}\right)}\right\}
$$

When Taylor vortexes appear in the flow, there is an increase in the torque. Stuart (1958) studied the torque in this flow regime and found the following relation for the torque coefficient:

$$
C_{M, l v}=\frac{1}{R e_{\theta}}\left[4+5.79\left(1-\frac{T a_{c}^{2}}{R e_{\theta}^{2} s_{P} / R_{i}}\right)\right]
$$

This equation is valid only for concentric cases.

For turbulent flows, Schilichting (1968) proposed that the torque coefficient is proportional to the Taylor number:

$$
C_{M, t}=k_{\varepsilon, t} T a^{-0.2}
$$

Table 1. Coefficients to be used with Eq. (9) for different eccentricities (after Nakabayashi et al. (1972)).

\begin{tabular}{|c|c|c|c|c|}
\hline \multirow{2}{*}{} & \multicolumn{4}{|c|}{$\boldsymbol{\varepsilon}$} \\
\cline { 2 - 5 } & $\mathbf{0}$ & $\mathbf{0 . 2 5}$ & $\mathbf{0 . 5 0}$ & $\mathbf{0 . 7 5}$ \\
\hline $\mathbf{k}_{\varepsilon, \mathbf{t}}$ & 0.02524 & 0.02904 & 0.03788 & 0.04920 \\
\hline
\end{tabular}

Experimental data obtained in the work of Nakabayashi et al. (1972) confirms this relationship. Based on his experimental data, Nakabayashi et al. (1972) calculated the proportionality coefficient to be used with Eq. (9) for different eccentricities as Tab. (1) shows.

\section{GENERALIZED CORRELATION FOR FRICTION COEFFICIENT}

In the laminar regime, Equation (2) together with Eq. (5) will give the correct values for concentric and eccentric cases. For turbulent flow, a generalized correlation for friction and coefficient was developed based on the correlations found in literature. The development shown below assumed narrow channels and moderate angular velocities because multiphase screw-pumps have inner to outer radii ratios higher than 0.99 and are expected to work with angular velocities lower than $5000 \mathrm{rpm}$. In this case, centrifugal forces due to rotation of inner cylinder will not be significant.

Among all the correlations for smooth turbulent flow, Eq. (3) proposed by Suzuki (1929) was considered the most adequate. In spite of Eqs (3) and (4) have similar results for turbulent flow; Eq. (3) has 
the parameter $\beta$ that can be adjusted. Therefore, with the correct adjustment of $\beta$, Eq. (3) can be used in the laminar with vortexes region even though it was developed for smooth turbulent cases.

Using experimental data from Yamada (1962), parameter $\beta$ was curve fitted as follows:

$\beta= \begin{cases}A \operatorname{Re}_{\theta}^{B}+C e^{\left(D / R e_{\theta}\right)} \quad\left(\operatorname{Re}_{\theta} \leq 4000 e \operatorname{Re}<2000\right) & (10) \\ 0.1713 * R e^{0.288}-1,7 e^{\left(-10410 / R e_{\theta}\right)} \text { (other ranges) }\end{cases}$

$A=\left\{\begin{array}{l}2.62 \times 10^{-2} R e^{2}-8.08 \times 10^{-1} R e+18.43(R e<200) \\ 2.64 \times 10^{-3} R e^{2}-3.20 \times 10^{-1} R e+7990(\operatorname{Re} \geq 200)\end{array}\right.$

$B=\left\{\begin{array}{l}4.40 \times 10^{-6} R e^{2}-2.92 \times 10^{-3} \operatorname{Re}-0.49(\operatorname{Re}<200) \\ -7.67 \times 10^{-8} R e^{2}+1.29 \times 10^{-4} \operatorname{Re}-1.17(\operatorname{Re} \geq 200)\end{array}\right.$

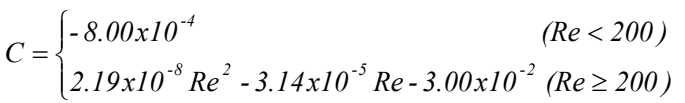

$D=\left\{\begin{array}{lc}32.77 R e+1608 & (\operatorname{Re}<200) \\ 4.14 \times 10^{-4} \operatorname{Re}^{2}+2.67 R e+3923 & (\operatorname{Re} \geq 200)\end{array}\right.$

Also the coefficient of Eq. (3) was altered so that this equation will reduce to Blasius equation when inner cylinder is not rotating:

$$
\begin{aligned}
\lambda_{s} & =\frac{0.3354}{\operatorname{Re}^{1 / 4}} \frac{1}{2}\left\{\left[1+0.629\left(\frac{\omega R_{i}}{U_{z}}\right)^{2}\right]^{3 / 8}\right. \\
& \left.+\left[1+0.629\left(\beta \frac{\omega R_{i}}{U_{z}}\right)^{2}\right]^{3 / 8}\right\}
\end{aligned}
$$

Figure (5) shows the results of this equation compared to experimental data of several authors. The results show sactisfactory agreement between predicted and experimental data. In Fig. (5a), where several geometries were studied, it is possible to realize that calculated data fit better data for narrower channels. This is expected since parameter $\beta$ in Eq. (10) was obtained for these geometries.

With these sactisfactory results, Eq. (15) can be re-written in the following manner:

$$
\begin{gathered}
\lambda_{s}=k_{a n} k_{r o t} \frac{0.3164}{R e^{I / 4}} \\
k_{a n}=1.06 \\
k_{r o t}=\frac{1}{2}\left\{\left[1+0.629\left(\frac{\omega R_{i}}{U_{z}}\right)^{2}\right]^{3 / 8}\right. \\
\left.+\left[1+0.629\left(\beta \frac{\omega R_{i}}{U_{z}}\right)^{2}\right]^{3 / 8}\right\}
\end{gathered}
$$

Equation (16), therefore, can be considered as the Blasius equation with two correction factors: $k_{a n}$, because the flow is annular, and $k_{\text {rot }}$, due to rotation of inner cylinder. Considering the fact that Blasius equation and the universal law developed by Prandtl give similar results when Reynolds number is not extremely high $\left(\operatorname{Re} \leq 10^{5}\right)$, the same correction factors could be applied to this equation, as follows:

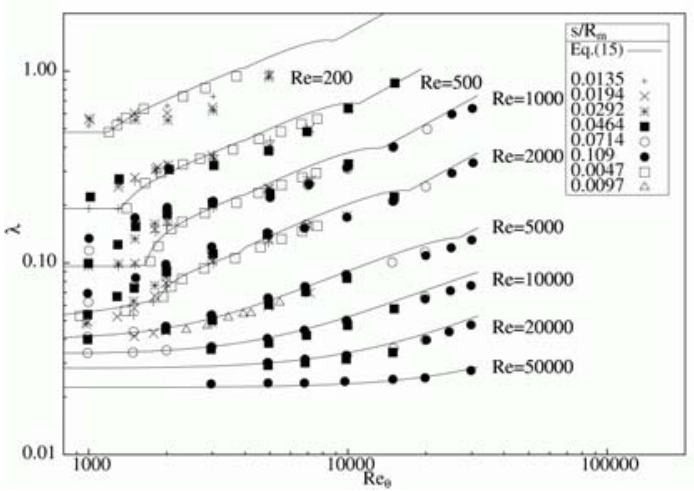

(a) Yamada (1962)

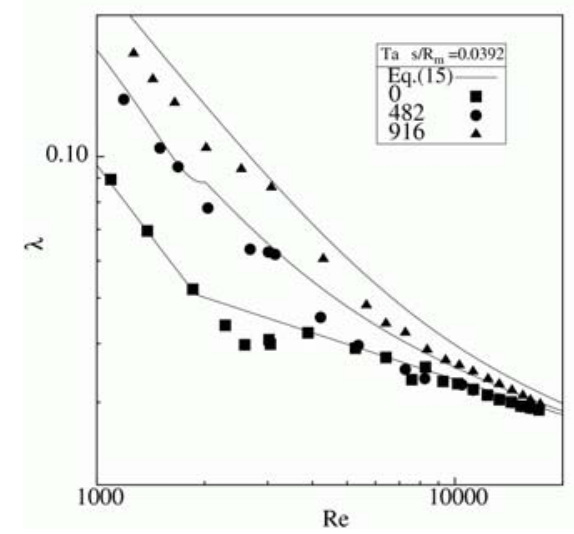

(b) Salhi et al (1992)

Figure 5. Comparison of Eq. (17) with experimental data from several authors.

$$
\frac{1}{\sqrt{\frac{\lambda_{s}}{k_{a n} k_{r o t}}}}=2 \log \left(\operatorname{Re} \sqrt{\frac{\lambda_{t}}{k_{a n} k_{r o t}}}\right)-0.8
$$

The next step to determine a general correlation is the calculation of the friction coefficient in the rough turbulent region. For this case, no correlation could be found in literature. Therefore, the same procedure used by Yamada (1962) was used. The only difference is the exponent of the power law, which was changed to $1 / 5$ following Schilichting (1968). The result, written in terms of correction factors as in Eq. (19), is given by eq. (20).

$$
\lambda_{t, r}=k_{a n} k_{r o t, r} \frac{1}{\left(2 \log \left(\frac{s_{p}}{k_{s}}\right)+1.74\right)^{2}}
$$




$$
k_{r o t, r}=\left[1+\left(\frac{5}{12}\right)^{2}\left(\frac{\omega R_{i}}{U_{z}}\right)^{2}\right]^{0.5}
$$

Assuming that the transition between smooth and rough turbulent flows will occur in way similar to the transition given by the Colebrook and White equation, Equations (19) and (20) can be combined as shown in Eq. (22).

$$
\begin{aligned}
& \frac{1}{\sqrt{\lambda_{t}}}=\frac{1.74}{\sqrt{k_{a n} k_{r o t, r}}}-2 \log \left[\left(\frac{k_{s}}{s_{p}}\right)^{\frac{1}{\sqrt{k_{a n} k_{r o t, r}}}}+\right.
\end{aligned}
$$

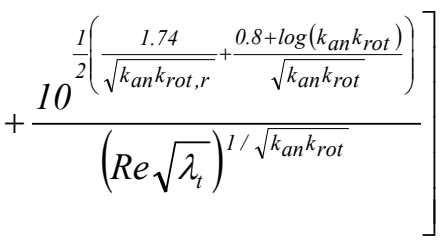

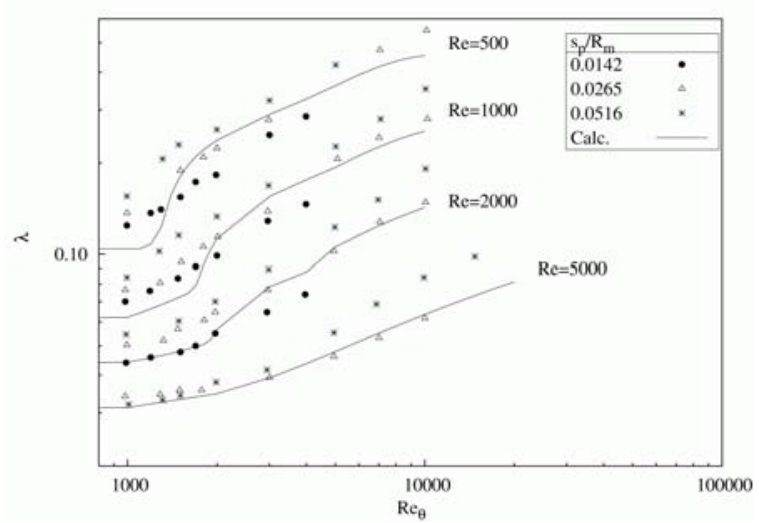

(a) $\varepsilon=0.25$

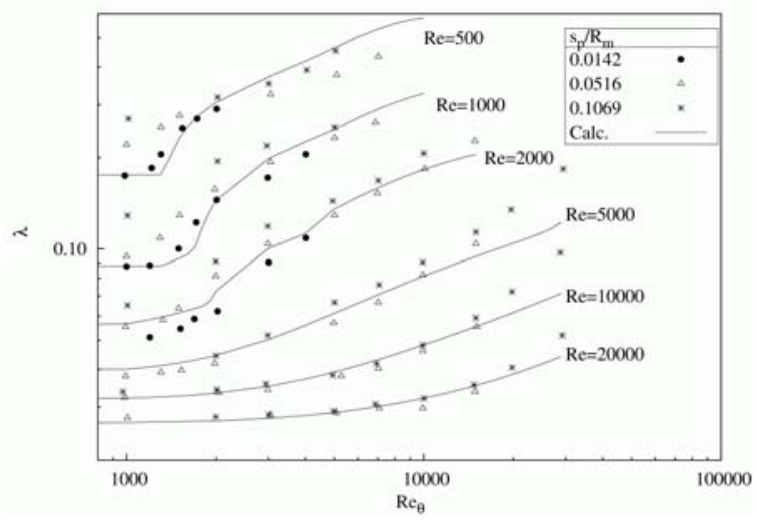

(b) $\varepsilon=0.75$

Figure 6. Comparison of Eq. (17) with experimental data from several authors.

Eccentricity effects can be included if it is assumed that it influences rotating and static channels in the same way. Therefore, correction factors can be calculated by Eq. (6), which can be represented by polynomials for smooth and rough turbulent flows:

$$
\begin{gathered}
k_{\varepsilon}=0.237 \varepsilon^{3}-0.6314 \varepsilon^{2}+0.0132 \varepsilon+1.0 \\
k_{\varepsilon, r}=-0.2577 \varepsilon^{2}-0.0561 \varepsilon+1.0
\end{gathered}
$$

And the general equation for smooth or rough turbulent flows in concentric or eccentric cases will be given by:

$$
\begin{aligned}
& \frac{1}{\sqrt{\lambda_{\varepsilon, t}}}=\frac{1.74}{\sqrt{k_{a n} k_{r o t, r} k_{\varepsilon, r}}}-2 \log \left[\left(\frac{k_{s}}{s_{p}}\right)^{\frac{1}{\sqrt{k_{a n} k_{r o t, r} k_{\varepsilon, r}}}}+\right.
\end{aligned}
$$

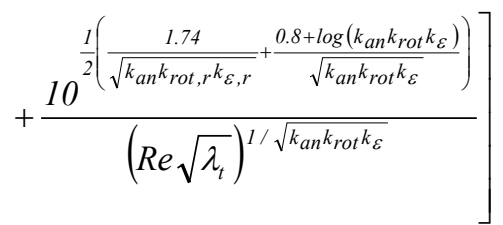

Figure 6 show the results of Eq. (25) compared to experimental data of Yamada, Nakabayashi and Maeda (1969), which studied eccentric rotating channels. The agreement between experimental and calculated data is good. Experimental results for rough turbulent flow were not found for comparison.

\section{GENERALIZED CORRELATION FOR TORQUE COEFFICIENT}

The torque coefficient in the laminar flow can be given by Eq. (7). When the flow is not laminar, however, the axial flow will increase the torque coefficient. Although no study about the torque coefficient with axial flow could be found, the same methodology used by Suzuki (1929) can be applied to calculate tangential losses. Suzuki (1929) added the two velocity components assuming the $1 / 7^{\text {th }}$ power law and used the resultant velocity to calculate shear stresses at the wall. The axial component of the shear stresses is used to calculated pressure drop as shown in Eq. (3) and, therefore, the tangent component could be used to calculate tangential losses represented by the torque coefficient. Using this approach, an expression for the torque coefficient for smooth turbulent flow can be deduced:

$$
\begin{aligned}
C_{M, s} & =\frac{0.045}{\operatorname{Re}_{\theta}^{0.25}}\left\{\left[1.592\left(\frac{R e_{z}}{2 R e_{\theta}}\right)^{2}+1\right]^{\frac{3}{8}}+\right. \\
& \left.+\beta\left[1.592\left(\frac{R e_{z}}{2 R e_{\theta}}\right)^{2}+\beta^{2}\right]^{\frac{3}{8}}\right\}
\end{aligned}
$$

Where the parameter $\beta$ is the same of Eq. (3). Similarly to Eq. (15), Eq. (26) can be expressed in terms of correction factors:

$$
C_{M, s}=k_{\theta}^{\prime} k_{r o t}^{\prime} \frac{0.3164}{\operatorname{Re}_{\theta}^{0.25}}
$$




$$
\begin{gathered}
k_{\theta}^{\prime}=1 / 7 \\
k_{r o t}^{\prime}=\left\{\left[1.592\left(\frac{R e_{z}}{2 R e_{\theta}}\right)^{2}+1\right]^{\frac{3}{8}}+\right. \\
\left.+\beta\left[1.592\left(\frac{R e_{z}}{2 R e_{\theta}}\right)^{2}+\beta^{2}\right]^{\frac{3}{8}}\right\}
\end{gathered}
$$

For rough turbulent flow, the same approach used to deduce Eq. (21) will give the following expression:

$$
\begin{gathered}
C_{M, r}=k_{\theta}^{\prime} k_{r o t, r}^{\prime} \frac{1}{\left(2 \log \left(\frac{s_{p}}{2 k_{s}}\right)+1.74\right)^{2}} \\
k_{r o t, r=}^{\prime}\left[1+\left(\frac{12}{5}\right)^{2}\left(\frac{U_{z}}{\omega R_{i}}\right)^{2}\right]^{0.5}
\end{gathered}
$$

The correction of torque coefficient due to eccentricity for the smooth turbulent flow through experimental data of Nakabayashi et al (1972) is shown in Tab. (1). Using their data a curve was fitted assuming that the increase of of torque coefficient with eccentricity has a similar behavior of the increase in laminar flow shown in Eq. (7). The correction factor due to eccentricity found with the curve fit is given by Eq. (31).

$$
k_{\varepsilon}^{\prime}=\frac{C_{M, s}}{C_{M, s \varepsilon}}=\frac{2\left(1+2 \varepsilon^{1.85}\right)}{\left(1-\varepsilon^{8.3}\right)^{1 / 2}\left(2+\varepsilon^{3.74}\right)}
$$

For the laminar with vortexes regime, no correction for the eccentricity was found. Experimental data from Nakabayashi et al (1972), however, evidences presence of Taylor vortexes is delayed by the eccentricity as shown in Fig. (7). For the laminar with Taylor vortexes regime, is assumed that the Taylor vortexes will appear in the flow at a fixed value for torque coefficient, which is approximately true as can be seen in Fig. (7). Using this approximation, it is possible to calculate the delayed Reynolds number using Eq. (7). The influence of Taylor vortexes becomes lower as eccentricity increases however it was not possible to take this fact into account. Therefore, the influence of vortexes in the laminar with vortexes region is considered the same $\left(k_{\varepsilon}^{\prime}=1\right)$ for all eccentricities. Using the correction factors (Eqs (28), (29), (30) and (32)) and assuming that the influence of eccentricity is the same either in smooth or rough turbulent flows, it is possible to define a correlation similar to Eq. (25) as follows:

$$
\begin{aligned}
& \frac{1}{\sqrt{C_{M, t}}}=\frac{1,74}{\sqrt{k_{\theta} k_{r o t, r} k_{\varepsilon}}}-2 \log \left\{\left(\frac{k_{s}}{2 s_{p}}\right)^{\frac{1}{\sqrt{k_{\theta} k_{r o t, r} k_{\varepsilon}}}}+\right. \\
& \left.+\frac{10^{\frac{1}{2}\left(\frac{1,74}{\sqrt{k_{\theta} k_{r o t, r} k_{\varepsilon}}}+\frac{0,8+\log \left(k_{\theta} k_{\text {rot }}\right)}{\sqrt{{ }^{\prime} k_{\text {rot }} k_{\varepsilon}}}\right)}}{\left(\operatorname{Re}_{\theta} \sqrt{C_{M, t}}\right)^{1 / \sqrt{k_{\theta} k_{\text {rot }} k_{\varepsilon}}}}\right\}
\end{aligned}
$$

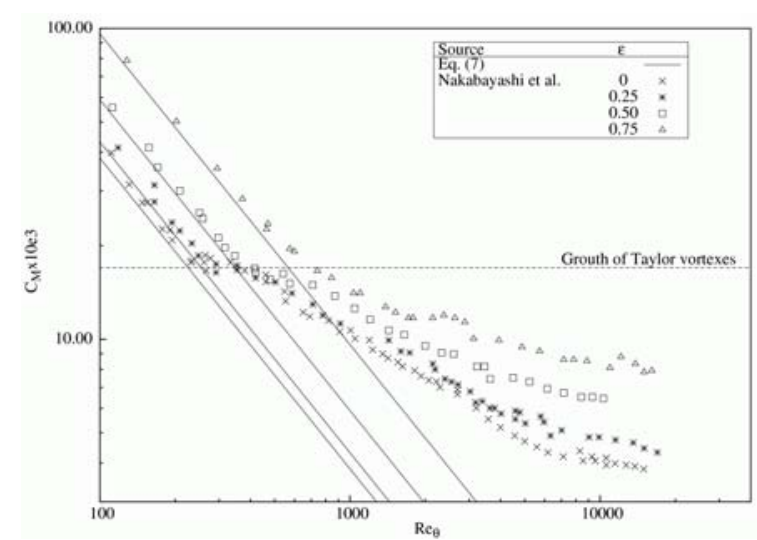

Figure 7. Influence of eccentricity over torque coefficient in the laminar with Taylor vortexes region.

Figure (8) shows a comparison between this equation and experimental data from Stuart (1958) and Nakabayashi et al (1972). The results show a sactisfactory agreement for the concentric case. When the channel is eccentric, the agreement is good except for the region where Taylor vortexes appear. In this region, characterized by a sudden increase in the torque coefficient, there is a significant deviation.

\section{CONCLUSIONS}

Two generalized correlations for friction and torque coefficients were developed in this paper. These correlations are valid for laminar with vortexes, smooth and rough turbulent flows. Together with equations for laminar flow proposed by other authors (Eqs. (2), (5) and (7)), it is possible to evaluate axial and tangential losses in narrow annular channels with inner cylinder rotating at moderate velocities (0-5000 rpm). The proposed correlations were compared to experimental results found in literature. The results for friction coefficient have a good agreement for concentric and eccentric cases in either smooth turbulent or laminar with vortexes regimes. When results for torque coefficient were compared, agreement is good in for concentric cases. If there is eccentricity, the proposed correlation overestimates the torque coefficient in the laminar with vortexes region. No experimental data for comparison were found in the rough turbulent regime. 


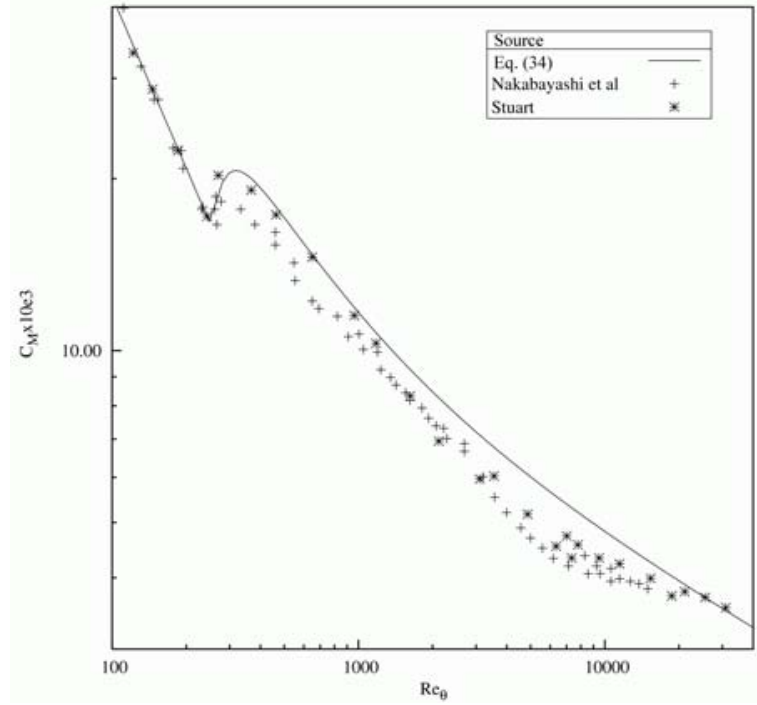

(a) $\varepsilon=0$

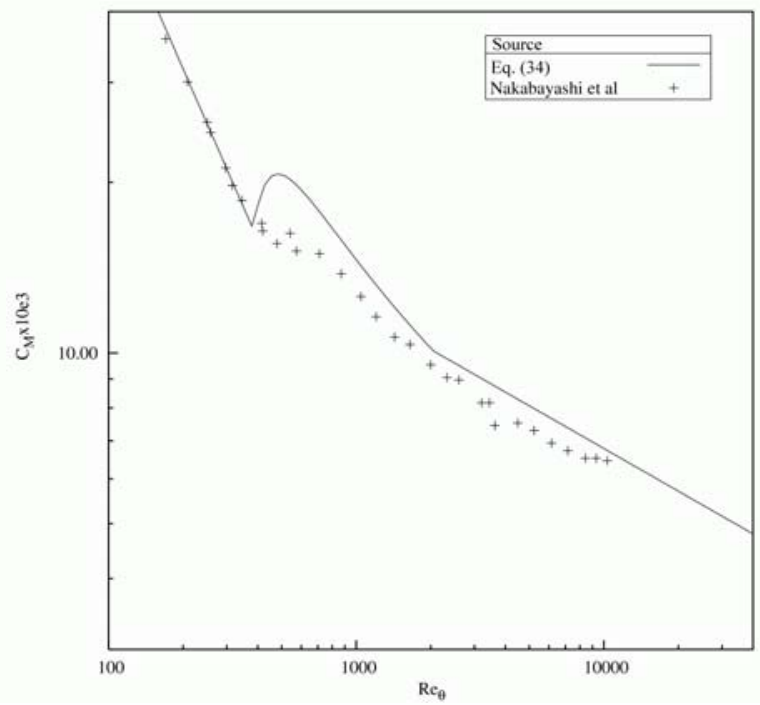

(b) $\varepsilon=0,50$

Figure 8. Comparison between calculated and experimental data from Stuart (1958) and Nakabayashi et al (1972).

\section{ACKNOWLEDGEMENT}

Authors are thankful to Petrobras, Curtiss Wright and Leistritz AG for the technical support and to FINEP and Petrobras for the financial support.

\section{REFERENCES}

Caetano, E. F., Shoham, O. and Brill, J. P., 1992, Upward Vertical Two-Phase Flow Through an Annulus - Part I: Single Phase Friction Factor, Taylor Bubble Rise Velocity and Flow Pattern Prediction, Journal of Energy Resources Technology Transactions of ASME, Vol. 114, No. 1, pp. 1-13.

Childs, D. W., 1983, Finite-Length Solutions for Rotordynamic Coefficients of Turbulent Annular Seals, Journal of Lubrication Technology -Transactions ASME, Vol. 105, No. 3, pp. 437-445.

Colebrook, C. F., 1939, Turbulent Flow in Pipes With Particular Reference to The Transition Region Between Smooth And Rough Pipe Laws, Journal of The Institution of Civil Engineers, Vol. 11, pp. 139-156.

Diprima, R. C. and Stuart, J. T., 1972, Flow between Eccentric Rotating Cylinders, Journal of Lubrication Technology, Vol. 94, pp. 266-274.

Escudier, M. P., Gouldson, I. W.; Oliveira, P. J. and Pinho, F. T., 2000, Effects of Inner Cylinder Rotation on Laminar Flow of a Newtonian Fluid through an Eccentric Annulus, International Journal of Heat and Fluid Flow, Vol. 21, No. 1, pp. 92-103.

Gunn, D. J. and Darling, C. W. W., 1963, Fluid Flow and Energy Losses in Non Circular Conduits, Transactions of the Institution of Chemical Engineers, Vol. 41, pp. 163-173.

Hirs, G. G., 1973, A Bulk Flow Theory for Turbulence in Lubricant Films, Journal of Lubrication Technology -Transactions ASME, Vol. 95, No. 2, pp. 137-146.

Idelchik, I. E. , 1994, Handbook of Hydraulic Resistence, 3rd Edition, Boca Raton, FL : CRC Press.

Kaye, J. and Elgar, E. C., 1957, Modes of Adiabatic and Diabatic Fluid Flow in an Annulus with an Inner Rotating Cylinder, Transactions of the American Society of Mechanical Engineers, Vol. 80, No. 3, pp. 753-765.

Nakabayashi, K., Yamada, Y., Mizuhara, S. E and Hiraoka, K., 1972, Viscous Frictional Moment and Pressure Distribution Between Eccentric Rotating Cylinders, when Inner Cylinder
Rotates, Transactions of the Japan Society of Mechanical Engineers, Vol. 38, No. 312, pp. 2075-2084.

Nakashima, C. Y., Oliveira Jr., S. and Caetano, E. F., 2002, Thermodynamic Model of a Twin-Screw Multiphase Pump, ASME Engineering Technology Conference on Energy, Paper ETCE2002/PROD-29164, February 4-6,2002, Houston, TX.

Nouri, J. M. and Whitelaw, J. H., 1997, Flow of Newtonian and non-Newtonian fluids in an eccentric annulus with rotation of the inner cylinder, International Journal of Heat and Fluid Flow, Vol. 18, No. 2, pp. 236-246.

Ooms, G. and Kampman-Reinhartz, B. E., 1996, Influence of drill pipe rotation and eccentricity on pressure drop over borehole during drilling, European Journal of Mechanics, B/Fluids, Vol. 15, No. 5, pp. 695-711.

Salhi, A., Rey, C. and Rosant, J. M., 1992, Pressure Drop in Single Phase and Two Phase Couette Poiseuille Flow, Journal of Fluids Engineering - Transactions of ASME, Vol. 114, No. 1, pp. 80-84.

Schlichting, H., 1968, Boundary Layer Theory. McGrawHill Book Company.

Stuart, J. T., 1958, On the Non-Linear Mechanics of Hydrodynamic Stability, Journal of Fluid Mechanics, Vol. 4, pp.121.

Suzuki, S., 1929, On the Leakage of Water Through Clearance Space, Journal of the Faculty of Engineering, Tokyo Imperial University, Vol. 18, No. 2, pp. 1-10.

Tao, L. N. and Donovan, W. F., 1955, Through-Flow in Concentric and Eccentric Annuli of Fine Clearance With and Without Relative Motion of the Boundaries, Transactions of the American Society of Mechanical Engineers, Vol. 77, pp. 12911301.

Wincek, M., 1992, Contribution for the Calculation of the Transport Behavior of Screw-Pumps Transporting Liquid-Gas Mixtures, Ph.D. Thesis (Portuguese translation by Adriana B. Costa), Friedrich-Alexander University of Erlangen-Nürnberg, Erlangen-Nürnberg, Germany.

Yamada, Y., 1962, Resistance of Flow Through an Annulus with an Inner Rotating Cylinder, Bulletin of the JSME, Vol. 5, No. 18, pp. 302-310.

Yamada, Y., Nakabayashi K. and Maeda, K., 1969, Pressure Drop Measurements of the Flow Through Eccentric Cylinders With Rotating Inner Cylinders, Bulletin of the Japan Society of Mechanical Engineers, Vol. 13, No. 53, pp. 1032-1040. 